\title{
Study of soft photon yield in pp and AA interactions at JINR
}

\author{
Elena Kokoulina ${ }^{1,2, \star}$, Nurlan Barlykov ${ }^{1}$, Vladimir Dudin ${ }^{1}$, Vladimir Dunin ${ }^{1}$, Andrey Kutov ${ }^{3}$, \\ Vladimir Nikitin ${ }^{1}$, Vasilii Riadovikov ${ }^{4}$, and Roman Shulyakovsky ${ }^{5}$ \\ ${ }^{1}$ Joint Institute for Nuclear Research 6 Joliot-Curie St 141980 Dubna, Moscow region, Russia \\ ${ }^{2}$ Sukhoi State Technical University of Gomel Prospect Octiabria, 48, 246746 Gomel, Belarus \\ ${ }^{3}$ Institute of Physics and Mathematics Komi SC UrD RAS Kommunisticheskaja st., 24, 167000 Syktyvkar, \\ Russia \\ ${ }^{4}$ Institute of High Energy Physics Science sq. 1, 142281 Protvino, Russia \\ ${ }^{5}$ Institute of Applied Physics Akademicheskaya str. 16, 220072 Minsk, Belarus
}

\begin{abstract}
Over 30 years there has been no comprehensive understanding of the mechanism of soft photons (energy smaller than $50 \mathrm{MeV}$ ) formation. Experimental data indicate an excess of their yield in hadron and nuclear interactions in comparison with calculations performed in QED. For a more thorough study of this phenomenon at the Nuclotron (a superconducting accelerator in JINR), preliminary measurements have been carried out with using an electromagnetic calorimeter based on BGO crystals. These results are consistent with the world data. In JINR, in connection with the building of a future accelerator complex NICA, it has become possible to carry out such studies in $p p, p \mathrm{~A}$ and AA interactions at energies up to $25 \mathrm{~A} \mathrm{GeV}$. Our group develops the conception of an heterogeneous electromagnetic calorimeter as "spaghetti" and "shashlik" types based on gadolinium gallium garnet (GaGG) crystals with a low threshold for registration of photons. The first tests of prototypes of them manufactured at JINR on the basis of the GaGG and a mixture of tungstate and copper as an absorber are reported.
\end{abstract}

\section{Introduction}

We call soft photons $(\mathrm{SPh})$ such photons that are the direct products of high energy interactions. They are not decay products of secondary particles and their energy is smaller than $50 \mathrm{MeV}$. More than thirty years ago the excess yield of SPh has been observed [1]. The following studies of this phenomenon have confirmed the excess yield in hadron and nuclear interactions from low up to high energies [2-6]. The excess gets 4-8 fold surplus for reactions with secondary charged particles. The unexpected result has been obtained in $e^{+} e^{-}$annihilation at DELPHI setup [6]. The excess of SPh yield depending on neutral particle multiplicity exceeds the theoretical estimation seventeen times over at 6 neutral pions. Such excess was observed only in the hadron channels. It was absent in the lepton channel: $e^{+} e^{-} \rightarrow \mu^{+} \mu^{-}$. The existing theoretical calculations based on the quantum electrodynamics can not predict and explain this excess. Physics only offer various phenomenological models taking into account quantum chromodynamics and experimental data.

\footnotetext{
^e-mail: kokoulina@jinr.ru
} 
Why are we interested in SPh? First of all, up to now, the nature of SPh remains obscure. Apparently, they are formed in the region of non perturbative quantum chromodynamics. So physicists build phenomenological models. Among them, the model of P. Lichard and L. Van Hove [7] is the most successful. It is based on hypothesis of the cold quark-gluon plasma (QGP) formation. This model supposes formation of a quark-gluon system which consists of a few quarks, antiquarks and gluons (about 40 partons). These partons are encountering with each other and reradiate soft photons because there is not enough of their energy to produce hadrons. At that, the main reactions are Compton scattering, $q+g \rightarrow q+\gamma$, and annihilation, $q+\bar{q} \rightarrow \gamma$. This model describes well the experimental yield of $\mathrm{SPh}$. There are also other phenomenological schemes explaining this yield $[8,9]$.

Our SVD-2 Collaboration develops the gluon dominance model (GDM) [10] in order to describe the region of high multiplicity (events with multiplicity way more than an average one). It takes into account two stages of multiparticle production (MP). In accordance to this model, at first stage, called quark-gluon cascade, a plentiful gluon splitting leads to abundance of gluons. We distinguish active and soft gluons. The active gluons have sufficiently high energy and can decay to quark-antiquark

pairs. Further, these pairs form secondary hadrons by recombination mechanism. This model is evidence the main sources of secondary hadrons are active gluons. But soft gluons can not split to quark pairs. So they are caught up by new formed hadrons and scattering on their valence quarks with formation of SPh. We have estimated the fraction of such gluons. It is about $50 \%$ of all gluons [11]. We could estimate the $\mathrm{SPh}$ emission region in the case of an almost equilibrium state using the black body emission spectrum in proton-proton interactions at the $\mathrm{U}-70$ accelerator with $70 \mathrm{GeV} / c$ proton beams. It represents a cube with side about 4-6 fm. This value exceeds the conventional region of hadronisation and raises some questions. Therefore, study of the soft gluon role will help understand deeper the mechanism of hadronisation.

Our experiment at U-70 was dedicated to study of the collective phenomena of secondary particles in events with high multiplicity. We managed to get the evidence of the Bose-Einstein condensate formation in this region [12]. Our Collaboration would like to answer question: Is there any connection between high multiplicity events and the excess yield of SPh.

And of course, the study of the soft gluon component will be very important for more detail description and understanding of the nucleon structure where an application of pQCD is hampered. We can not help but repeat the important words about them [13] : "Gluons are carriers of the strong force, bind quarks together inside nucleons and nuclei and generate nearly all of the visible mass in the universe. Despite their importance, fundamental questions remain about the role of gluons in nucleon and nuclei. These questions can only be answered with a powerful new electron ion collider (EIC) providing unprecedented precision and versatility." Xiangdong Ji recalled them at the "Confinement and hadron spectrum" conference in Ireland in 2018.

\section{First results of SPh study at Nuclotron}

Among with theoretical estimations of the SPh yield we carry out active experimental study of them in proton and nuclear collisions at Nuclotron (the JINR's setup) and plan extending them [14] at future accelerator center NICA [15]. This task was one of the main goals of Thermalization project that planned to solve at the U-70 accelerator. We could get down to this study at the ending of our project. Toward this end our Collaboration manufactured the electromagnetic calorimeter (ECal) on the base of BGO (bismuth ortogermanate) scintillators [16]. BGO crystals have a small radiation length $\mathrm{X}_{0}=1.12 \mathrm{~cm}$ that permits reducing considerably the volume of our device. Moreover, this scintillator has small sensibility to neutrons. It was important at the measurement of a gamma-radiation. At manufacturing of such calorimeter, the problems of uniform distributions of activator in the crystal volume did not appear. 

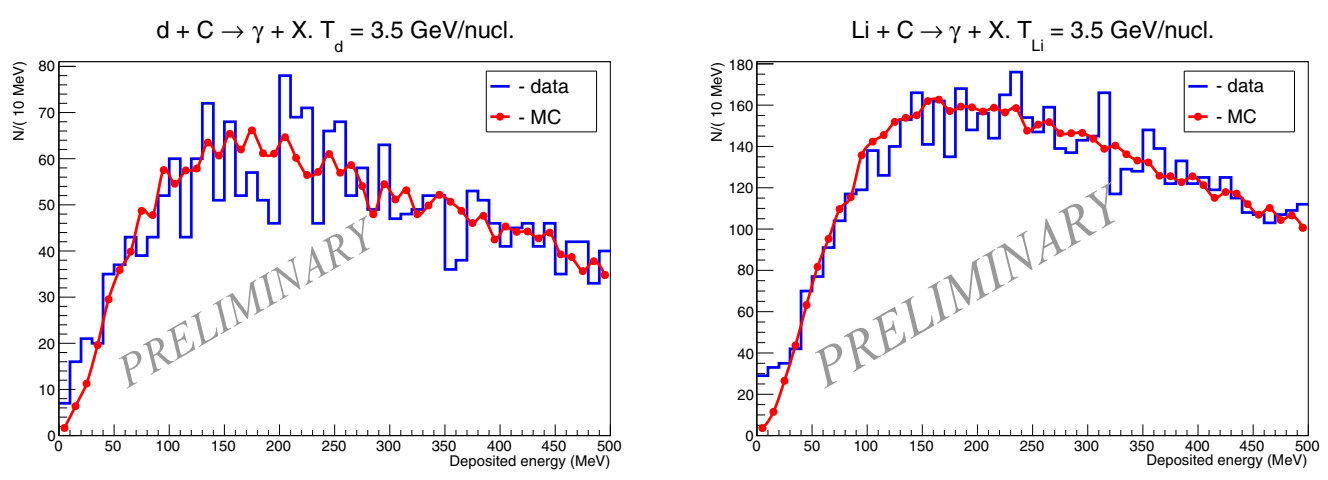

Figure 1. Energy release in BGO calorimeter with a pre-shower in $\mathrm{d}+\mathrm{C}$ (the left panel) and in $\mathrm{Li}+\mathrm{C}$ (the right panel) interactions at Nuclotron. Monte Carlo simulation (the red line) and data (the blue line).

This calorimeter presents a square matrix composed of $49(7 \times 7)$ counters. The counter with a demultiplier and a preliminary amplifier are placed directly on the panel of PMT, every crystal has the parallelepiped form, $30 \times 30 \times 180 \mathrm{~mm}^{3}$. $180 \mathrm{~mm}$ correspond to 16 radiation lengths. The lateral facets of crystals are wrapped up in Tyvic (the thickness $120 \mu \mathrm{m}$ ) for increasing of light gathering. The PMT 9106SB are used (ET Enterprises). They have 8 dynodes and high quantum efficiency in the green part of spectrum. PMT is glued to the crystal by the optic EPO-TEK 301 glue.

This calorimeter with counters is surrounded by the scintillator detectors of a guard veto-system. It was placed inside of the thermostat. The thermo stabilisation was realised by a cooling system Huber $006 \mathrm{~B}$. The temperature of liquid in the thermostat can vary from $-20^{\circ}$ up to $+40^{\circ} \mathrm{C}$. After a few trials the temperature has been chosen $+18^{\circ} \mathrm{C}$.

The plastic veto-detector of charged particles $\left(23 \times 23 \times 1 \mathrm{~cm}^{3}\right)$ was placed before the crystals and behind it an assembly of 4 plastics of a pre-shower $\left(18 \times 4.5 \times 1 \mathrm{~cm}^{3}\right)$ was installed. A lead $2 \mathrm{~mm}$ convertor was put between the front-veto and plastics. The trigger was produced by the signal from any 2 of 4 pre-shower counters. There were two large veto-counters in front of the target to forbid a response from the beam halo. This BGO calorimeter with veto-detectors has been laid at the Nuclotron hall at the NIS-GIBS setup.

During the Nuclotron run (2015 year) this BGO calorimeter was set at an angle of $16^{\circ}$ relative to the beam direction. The front plane of the crystals was away from the target at the distance $203 \mathrm{~cm}$. The digitisation of plastic scintillators was realised with a CAMAC ADCs (Lecroy 2249A) and TDCs (LeCroy 2228A), the digitisation of the analog signals of calorimeter - by ADC CC-008.

Monte Carlo simulation of this calorimeter has been carried out at the conditions of the last assembly and the beam energy - 3.5 AGeV. Monte-Carlo simulation (uRQMD+Geant-3.21) is used. Geant-4 has shown the same result. After data processing we have obtained SPh spectra of energy release in deuterium-carbon (Fig. 1, the left panel) and lithium-carbon (Fig. 1, the right panel) interactions. In the region of energy below $50 \mathrm{MeV}$, a noticeable excess over Monte-Carlo simulation has been observed. It agrees well to other SPh experiments [17].

\section{Simulation and testing of "spaghetti" and "shashlik" calorimeters}

To continue SPh study at JINR's setups we started designing a heterogeneous ECals with a low (about $10 \mathrm{MeV}$ ) threshold of registration [18]. At present, physicists use homogeneous, pure crystal ECals 

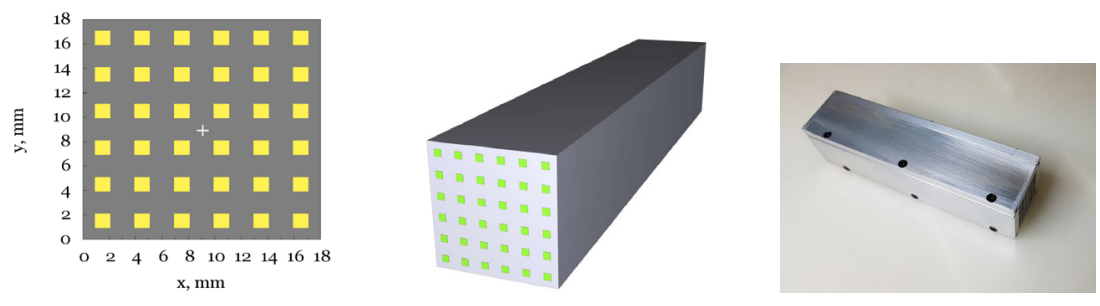

Figure 2. Scheme of the cell for "spaghetti" ECal and its assembly.

and heterogeneous, such types as "spaghetti" [19] and "shashlik" [18]. Physicists use the abridgment SpaCal saying about "spaghetti" ECal. Crystal ECals are very expensive. Their cost gets $50 \$ / \mathrm{cm}^{3}$. In the second case, we lose a little with the energy resolution but we gain with a cost which gets $5 \div 30$ $\$ / \mathrm{cm}^{3}$. We would like to occupy a niche between heterogeneous structures ("spaghetti" or "shashlik" type) with light yield $\sim 3-6 \mathrm{ph} / \mathrm{MeV}$ and crystal detectors with light yield $\sim 10,000-40,000 \mathrm{ph} / \mathrm{MeV}$ and tune them for the region $10-50 \mathrm{MeV}(\mathrm{SPh})$. It is known that the threshold for shashlik types currently used starts from $100 \mathrm{MeV}$ which is not suitable for our task. The main cause making an impact on such choice was using of heavy crystal in the capacity of scintillator.

We are aimed at the creation of "heavy" ECals (by analogy with heavy ions). We are going to substitute light scintillator material [20] for very dense (heavy) crystal with high specific light yield. It will allow us to build the device that is capable to keep the considerable compactness (space-saving about $30 \%$ ). To produce the "spaghetti" type we have chosen a mono-crystal of gadolinium-gallium garnet, $\mathrm{Gd}_{3} \mathrm{Al}_{2} \mathrm{Ga}_{3} \mathrm{O}_{12}: \mathrm{Ce}(\mathrm{GaGG})$, as a scintillator and tungsten+copper composite by way of the absorber. We wait the decay time $\sim 90 \mathrm{~ns}$; the light yield $\sim 45,000-55,000 \mathrm{ph} / \mathrm{MeV}$; price about 25-35 $\$ / \mathrm{cm}^{3}$ of volume, and good radiation resistance.

Comparison of properties of several crystals are presented in table 1. We choose crystal GaGG because it is a fast-acting scintillator, has a high light yield. The light yield in GaGG is 4 times more than in BGO. What is more, irradiation tests demonstrate good radiation resistance of GaGG. And we can acquire these crystals from the well-known domestic firm "Fomos-Materials" [21]. It has a long history, rich in scientific researches and technological developments that is competitive with the foreign analogues (and even surpass them) on an equal basis. Thanks to knowledge and professionalism of founding developers it was possible to obtain a material of high quality.

The first prototype of the detector cell $\mathrm{SpaCal}$ was an assembly of $\mathrm{W}+\mathrm{Cu}$ composite plates and GaGG: Ce rods, with the shape of a rectangular parallelepiped: $18 \times 18 \times 100 \mathrm{~mm}^{3}$. It had $6 \times 6$ $\left(1 \times 1 \times 100 \mathrm{~mm}^{3}\right)$ scintillator rods surrounded by absorber. The surfaces of plates and absorber rods were coated with a $10 \mu \mathrm{m}$ polymer dim white reflector. We tested two such assemblies. "Spaghetti" technology is a type of the sampling calorimeter with scintillation fibres running along a shower direction [19]. Granularity of whole module is defined by the granularity of read-out system. The scheme of a single cell and its view in an assembly is shown in Fig. 2.

In spring of 2019 year the experimental tests have been carried out by ourselves in Mainz (Mainz Microtron, Germany). Two manufactured cells were irradiated by 41, 51 and $69 \mathrm{MeV}$-photons. In Fig. 3, both Monte Carlo simulation of such a cell and our experimental result demonstrate the same behaviour in the case of $51 \mathrm{MeV}$-photon beam. It is seen that in the case of an even number of rods a narrow photon beam hitting in the centre of the cell turns out in the absorber plate. We are only observing the tail of the Gauss distribution. 
Table 1. Comparison of scintillator properties

\begin{tabular}{llll}
\hline parameters & $\mathrm{GaGG}$ & $\mathrm{Bi}_{4} \mathrm{Ge}_{3} \mathrm{O}_{12}$ & $\mathrm{NaI}: \mathrm{Tl}$ \\
\hline light yield, $10^{3} \mathrm{ph} / \mathrm{MeV}$ & 57 & 8 & 4.5 \\
energy resolution at $662 \mathrm{keV}$ & 5.2 & 12 & 7.1 \\
decay time, ns & 88 & 300 & 250 \\
density, g/cm3 & 6.63 & 7.13 & 3.67 \\
\hline
\end{tabular}
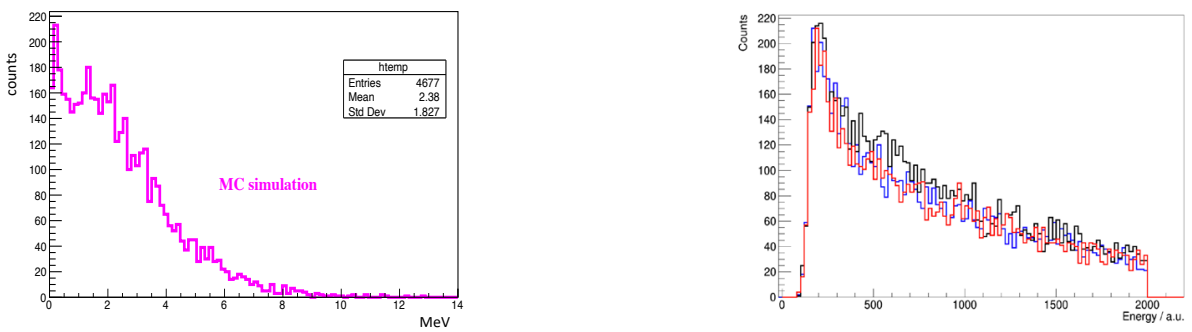

Figure 3. Energy release: Monte Carlo simulation and experimental data for $51 \mathrm{MeV}$-photon irradiation of SpaCal. The different colours of line correspond to a few small falling angles of a photon beam (the right panel)

To optimize the properties of ECals, modeling of both types has been carried out. We have opted for "spaghetti" simulation the following parameters: the matrix $25 \times 25$ GaGG crystal rods with $3 \times 3$ $\mathrm{mm}^{2}$ cross section, the ratio between the tungsten and copper components for the absorber was chosen 5:95. The total size of such a cell was equal to $101 \times 101 \times 150 \mathrm{~mm}^{3}$. The gap between rods consisted of $1 \mathrm{~mm}$. For "shashlik" we constrained of sixteen GaGG plates $\left(100 \times 100 \times 3 \mathrm{~mm}^{3}\right)$ and fifteen 2 -mm absorber plates with the same ratio of $\mathrm{W} / \mathrm{Cu}$ components and the same cross section of every plate like in the SpaCal case. The total thickness of that prototype consists of $78 \mathrm{~mm}$. In Fig. 4 the energy resolution of both types of ECals is presented. It is seen that at that choice of ECal parameters the "shashlik" type gives the energy resolution much better and can be used in our future experimental study.

\section{Conclusions}

The unique physical program of SPh study [14] can be carried out at accelerator setups of JINR or elsewhere. Simulation both "spaghetti" and "shashlik" types of heterogeneous ECals gives evidence that our program are quite reasonable and feasible. Their characteristics (the energy resolution and others) can be improved by changing of the cross-section of crystal rods, adjusting the absorber density, and optimising the length of ECals for the energy field of SPh. The energy resolution will be 10 and better for photons with energy above $50 \mathrm{MeV}$ with SiPM and the correct scheme for light transporting to a photo detector.

Simulation of "shashlik" type with GaGG as a scintillator and W/Cu as an absorber demonstrate the energy resolution at low energy much better than with "spaghetti" type. Our experimental tests at photon beams are in progress. We hope to carry out these investigations in this year. We are also learning possibilities of using of Glass and Glass Ceramic Stoichiometric and Gd3+ heavy loaded $\mathrm{BaO} * 2 \mathrm{SiO} 2 \mathrm{Ce}(\mathrm{DSB}: \mathrm{Ce}$ ) scintillation material for ECal application. We express our sincere gratitude 

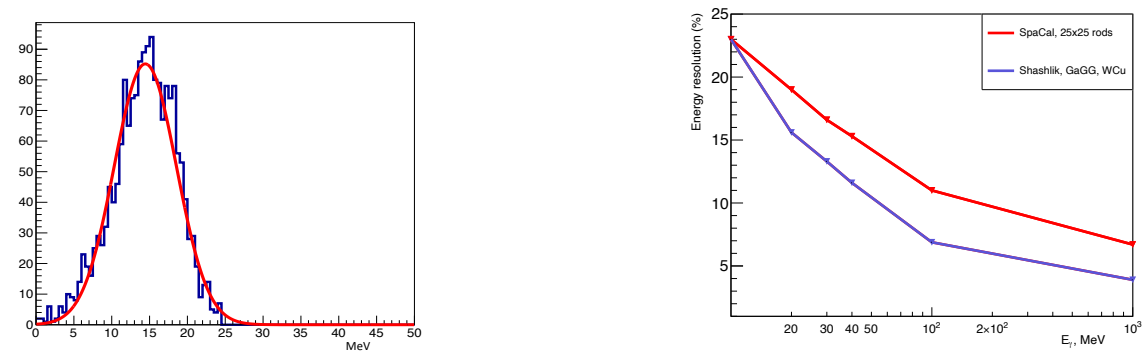

Figure 4. The left panel: spectrum of the energy release in "shashlik" $\left(\mathrm{E}_{\gamma}=30 \mathrm{MeV}\right)$. The right panel: the energy resolution in the"spaghetti" (the red line) versus the "shashlik" (the blue line) calorimeters.

to the SVD-2 Collaboration for the fruitful work in the manufacture of SpaCal, getting and discussing the main results.

\section{References}

[1] P.V. Chliapnikov et al., Physics Letters B141, 276-280 (1984)

[2] J. Schukraft. HELIOS Collaboration, Nuclear Physics A498, 79-92 (1989)

[3] S. Banerjee et al. SOPHIE/WA83, Physics Letters B305, 82-186 (1993)

[4] A. Belogianni et al. WA91, Physics Letters B408, 487-492 (1997)

[5] A. Belogianni et al. WA102, Physics Letters B548, 129-139 (2002)

[6] J. Abdallah et al., DELPHI Collaboration, Eur. Phys. J C47, 273-294 (2006)

[7] P. Lichard, L. Van Hove, Physics Letters B245, 605-608 (1990)

[8] S. Barshay, Physics Letters B227, 279-284 (1989)

[9] Wong Cheuk-Yin, Phys. Rev. Lett. C81, 064903 (2010)

[10] E. Kokoulina, Acta Phys. Polon. B35, 295-302 (2004)

[11] E.S. Kokoulina, Phys. Part. Nucl. Lett. 13, 74-78 (2016)

[12] Afonin, A.G. et al. SVD Collaboration EPJ Web of Conferences 37, 06002 (2013)

[13] Xiangdong Ji and Tsung-Dao Lee. Report at the XIIIth Quark Confinement and Hadron Spectrum Aug. 3, 2018, Maynooth University, Ireland

[14] V.V. Avdeichikov et al. NICA White Paper (JINR, Dubna, 2013) p.135

http://theor.jinr.ru/twiki-cgi/view/NICA/NICAWhitePaper

[15] V. Golovatyuk, et al. Eur.Phys.J. A52, 212 (2016)

[16] E.N. Ardashev et al. Instr. Exp. Tech. 58, 18 (2015)

[17] J. Schukraft. HELIOS Collaboration. Nucl.Phys. A498, 79 (1989)

[18] C. Grupen and B. Shwartz. Particles detectors (Cambridge University Press, New York, 2011) $652 \mathrm{p}$.

[19] D. Acosta et al. Nucl.Instrum.Meth. A294, 193 (1990)

[20] Paul Lecoq, Alexander Gektin, and Mikhail Korzhik. Inorganic Scintillators for Detector Systems (Second Edition. Springer, Berlin, 2017) 408 p.

[21] Nina S. Kozlova et al. Modern Electronic Materials 4, 7 (2018) 